\title{
Novel Endoscope System with Plasma Flushing for Off-Pump Cardiac Surgery
}

\author{
Ken Masamune ${ }^{1}$, Tetsuya Horiuchi ${ }^{1}$, Masahiro Mizutani ${ }^{1}$, Hiromasa Yamashita ${ }^{1}$, \\ Hiroyuki Tsukihara ${ }^{2}$, Noboru Motomura ${ }^{2}$, Shinichi Takamoto ${ }^{2}$, Hongen Liao ${ }^{3}$, \\ and Takeyoshi Dohi ${ }^{1}$ \\ ${ }^{1}$ Graduate School of Information Science and Technology, The University of Tokyo \\ ${ }^{2}$ Graduate School of Medicine, The University of Tokyo \\ ${ }^{3}$ Graduate School of Engineering, The University of Tokyo
}

masa@i.u-tokyo.ac.jp

\begin{abstract}
The purpose of this study is to develop a new endoscope for performing simple surgical tasks inside a cardiac atrium/chamber filled with blood, i.e., for performing "off-pump" cardiac surgeries. In general, it is very difficult to observe the inner wall of the vessels containing circulating blood because the light from the endoscope is scattered by the red blood cells. "Plasma flushing" performed using the separator system is developed to observe the inner side of the heart filled with blood and to remove blood cells from the front of the endoscope tip. The system was used in in vitro quantitative measurement of the device performance and in vivo experiments on a swine. In these experiments, we successfully obtained highresolution images of the interior of the heart during off-pump surgery.
\end{abstract}

\section{Introduction}

Currently, the cardiopulmonary bypass system is being widely used for performing cardiotomy under extracorporeal circulation even though the operation task is quite simple-the operation involves simply clipping valve leaflets, cutting or shortening a chord, closing the septal hole, etc. However, there are risks associated with the use of an artificial heart-lung machine during the operation. For example, complications such as cerebral infarct and pulmonary infarct may arise after operation. Thus, in the case of simple surgery inside the heart, off-pump surgery is ideal for increasing the quality of life of the patients.

Some research groups and companies have been trying to perform surgical tasks by using off-pumps, e.g., Linte CA[3] developed the augmented reality environment for off-pump mitral valve implantation, and L. Ming[4] developed an MRI-compatible robot for assisting off-pump artificial aortic valve replacement. Tomasz [1] and Evalve Inc.[2] reported the development of the surgical catheter device "Mitraclip" that can be used to perform off-pump edge-to-edge (E2E) repair for mitral regurgitation; in this procedure, the obstruction of transmitral flow is reduced by attaching clips to the leaflets of the mitral valve through catheter manipulation. However, it is very difficult to perform quick, efficient operations by adopting these procedures or using these devices because of the lack of image information during surgery. For each 
task, surgeons have to use low-resolution ultrasound images, 2D X-ray images obtained by X-ray exposure, or delayed MR images in order to observe the procedure during the surgery. The best observation method is to obtain an endoscopic view of the interior of the heart. Endovascular endoscopes are widely used not only for diagnosis but also for the surgical treatment of vessel stenosis, vessel sclerosis, etc. However, it is very difficult to observe the inner side of the vessels containing circulating blood because the light from the endoscope is scattered by the red blood cells and clear images are not obtained if saline flushing of these vessels is not performed.

To overcome the above problems, we developed a new endoscope system to acquire a clear endoscopic view that would help perform simple surgical tasks inside a cardiac atrium/chamber filled with blood. We utilized a "plasma flushing" system to remove blood cells from the front of the endoscope tip, as described in section 2. A new tip design is adopted for more efficient observation. In section 3, experiments and their results are described. In contrast, when using conventional endovascular endoscopes, an innovative method must be developed for observation of the inner surface of a wider heart chamber, given the strong turbulence caused by heart beats.

\section{Design and Development of New Endoscope}

To observe the inner chamber of the heart filled with blood, we developed a "plasma flushing" system to remove blood cells from the front of the endoscope tip. Figure 1 shows an overview of our prototype system. The system comprises a whole endoscope (The diameter and length of the image guide fiber are $3.8 \mathrm{~mm}$ and $200 \mathrm{~mm}$, respectively.) with a small channel $(\mathrm{d}=2.0 \mathrm{~mm})$; this channel comprises a coaxial

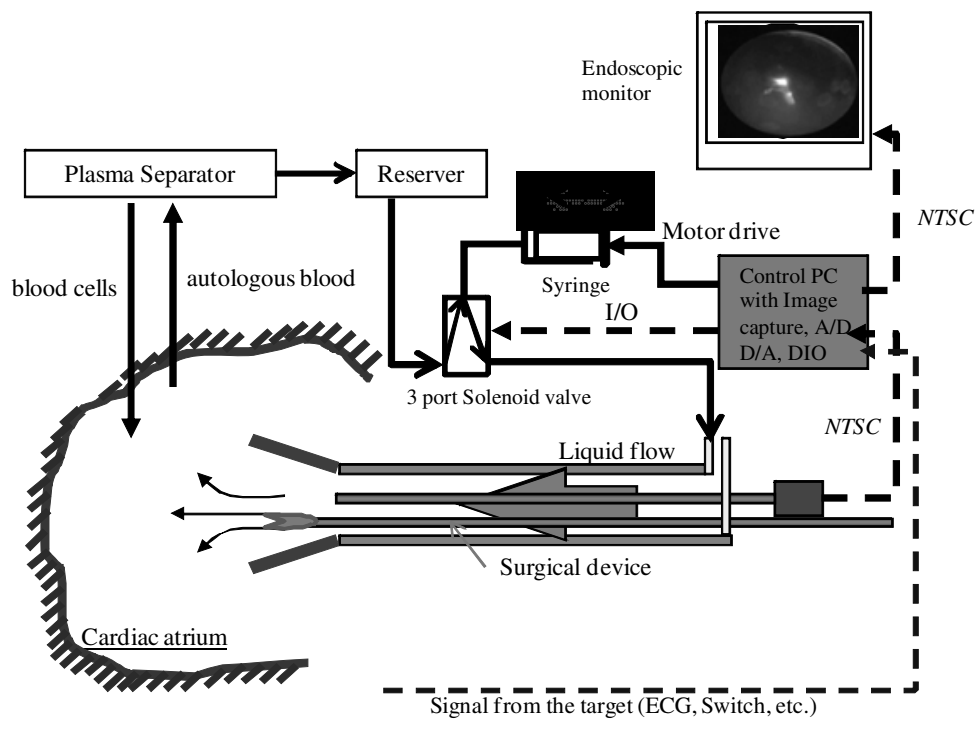

Fig. 1. Overview of the plasma flushing system: a fiber endoscope, surgical device, and the liquid flow are integrated inside the endoscope device $(\mathrm{d}=10 \mathrm{~mm})$ 
flush channel and a small channel for forceps; the system also comprises a three-port solenoid valve, a syringe with a pumping actuator, a control PC with an add-in board for capturing images, A/D, D/A, and DIO extension boards to control the timing of the flushing, and an observation monitor.

The required specifications for the endoscope are as follows:

1. The external diameter should be less than $10 \mathrm{~mm}$.

2. The cardiovascular function should not be disturbed.

3. Points up to $10 \mathrm{~mm}$ from the endoscope tip should be observable.

4. The endoscope must be biocompatible and sterilizable.

Figure 2 shows the prototype design of the device that satisfies the above requirements. Inside the outer pipe, a fiber-optic endoscope $(\mathrm{d}=0.7 \mathrm{~mm}$; AS-003, FiberTech Inc.) and biopsy forceps $(\mathrm{d}=1.7 \mathrm{~mm})$ are placed; further, the ends of the pipe are sealed by silicone rubber. Inside of the outer pipe, $d=0.7 \mathrm{~mm}$ fiber-optic endoscope (AS-003, FiberTech Inc.) and $d=1.7 \mathrm{~mm}$ biopsy forceps are set and the end of pipe are sealed by silicone rubber. A conventional endoscope can only be used to observe blood flow in a beating heart because blood contains corpuscles that reflect and scatter light. To observe intracardiac surgery, we used a clear liquid flushed from the endoscope tip created a transparent region to observe the object. We proposed the use of plasma from autologous blood as the flushing liquid, because it is transparent, viscous, fully biocompatible, and if the total amount of liquid can be controlled, it will not cause stress to the heart during the operation.

We attached the new tool called the "separator," which looks like an umbrella, to separate liquid and blood at the device tip. The separator is made from a thin plastic film and six frames that are made of SUS304. The frames are arranged in a radial pattern around the center of the endoscope. This separator can be folded by pushing the outer sheath, and it recovers its original shape by the action of a self-generated spring force.

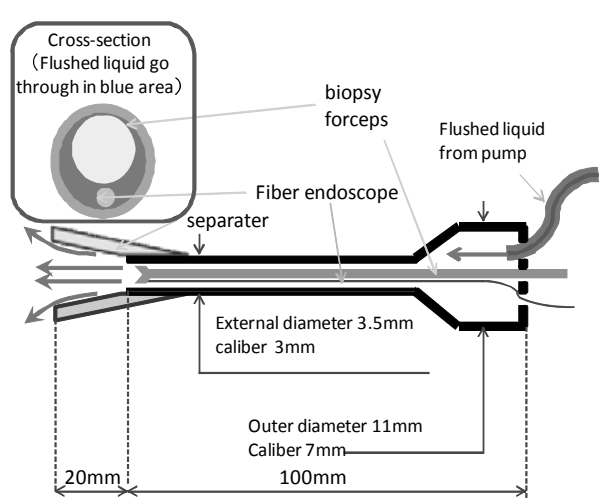

a)

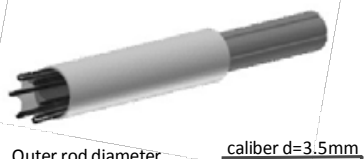

b)

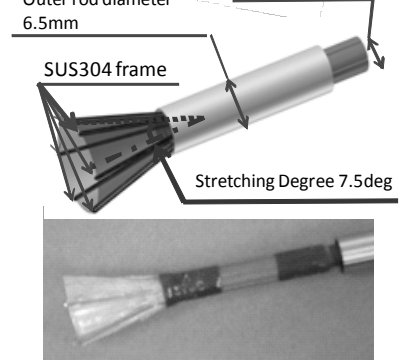

Fig. 2. Detailed design of the endoscopic device with separator tip: a) Status before device insertion b) Opening of the separator c) Developed separator tip 


\section{Experiments and Results}

\subsection{Evaluation Experiment for Observable Depth}

We performed a phantom experiment to evaluate the relationship between the angle of the separator of the tip and the observable depth. The experimental procedure is as follows:

1. Prepare a tank filled with blood and place a plastic phantom inside the tank.

2. Fix the endoscope device on an XYZ stage to measure the distance between the device tip and the phantom.

3. Measure the maximum depth upto which the phantom can be clearly observed.

4. Vary the following parameters: the separator open-angle, the distance, and the flushing speed.

a)

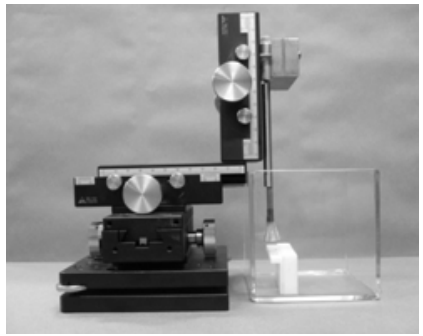

b)

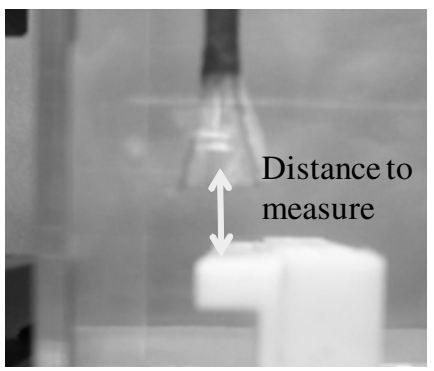

c)
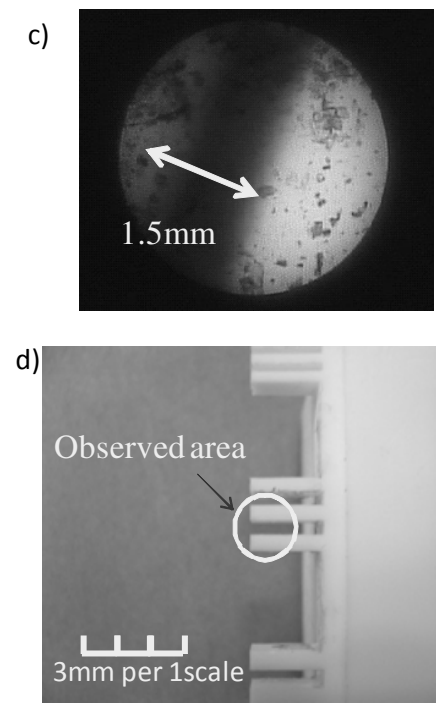

Fig. 3. Experimental setup: a) Endoscopic device attached on an XYZ stage along with the phantom; b) The distance to measure; c) Endoscope image obtained during flushing performed inside the blood pool; d) Observed area of the phantom

Table 1. Relationship between separator angle and measured observation depth

\begin{tabular}{|r|c|c|}
\hline $\begin{array}{c}\text { Separator } \\
\text { angle[deg] }\end{array}$ & $\begin{array}{c}\text { Flush } \\
\text { speed }[\mathbf{m l} / \mathbf{s}]\end{array}$ & $\begin{array}{c}\text { Observable } \\
\text { depth }[\mathbf{m m}]\end{array}$ \\
\hline $\mathbf{1 3}$ & 5.4 & 2.5 \\
\hline $\mathbf{7 . 5}$ & 2.4 & 3.8 \\
\hline $\mathbf{0}$ & 4.8 & 3.5 \\
\hline
\end{tabular}


In this experiment, we used glycerin as a flushing liquid instead of blood plasma.

The results are listed in Table 1. The listed flushing speeds are the maximum speeds required to observe the target phantom. The observable depth was the best when the open angle of the separator was set to $7.5^{\circ}$.

\subsection{Evaluation Experiment for Observation Area}

The observation area (visual field) was evaluated using a phantom along with a device that was equipped with a separator and one that was not equipped with a separator. In this experiment, the separator with an open angle of $7.5^{\circ}$ was used since it gave the best performance in the previous experiment. We used another phantom, shown in Figure 4, to measure the circular observation area with a diameter of $5 \mathrm{~mm}$. We evaluated the maximum depth by varying the distance between the endoscope tip and the phantom while the entire circle was in view.

The results are listed in Table 2. It was impossible to observe the phantom at any distance when the endoscope was used without a separator.

Table 2. Dependence of the maximum observable depth on the separator

\begin{tabular}{|c|c|c|}
\hline Separator & $\begin{array}{c}\text { Observation } \\
\text { depth [mm] }\end{array}$ & $\begin{array}{c}\text { Flushing } \\
\text { speed }[\mathbf{m l} / \mathbf{s}]\end{array}$ \\
\hline Present & 3 & 4 \\
\hline Absent & Not Visible & At any speed \\
\hline
\end{tabular}

a)

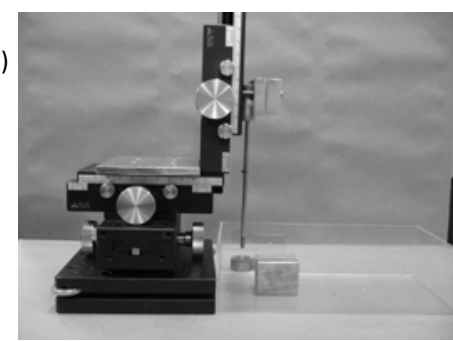

b)

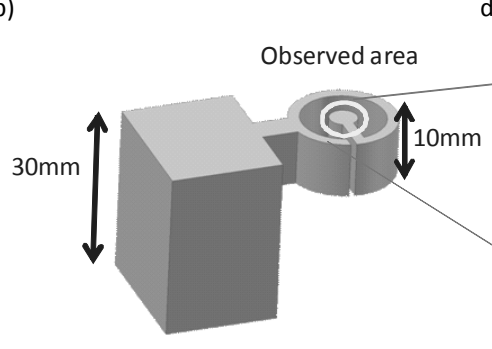

c)

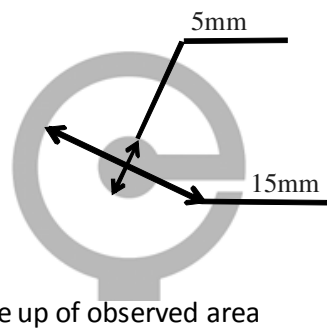

d)

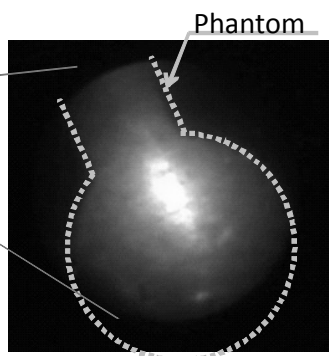

Fig. 4. Experimental setup: a) Endoscopic device attached on an XYZ stage and the phantom; b) Phantom for measuring the observable area; c) Dimensions of the phantom; d) Observed phantom during the flushing performed in the blood pool 


\subsection{In vivo Experiments and Results}

In vivo experiments were performed on a swine whose heart size is similar to the size of a human heart. In the experiment, the device was inserted into the right atrium through a trocar with a diameter of $4.0 \mathrm{~mm}$; the trocar was placed in an off-pump.

We chose the tip of tricuspid valve and chorda tendinea in the right atrium for observation. Since the anatomy of the swine heart is different from that of the human heart, the trocar-insertion approach from the left atrium is very severe. Thus, we chose the right atrium for the in vivo experiment. The observation was monitored by real-time ultrasound imaging, which was required to recognize the position of the device tip before performing the flushing in the right atrium. This is because a clear view was available in an extremely narrow space and the surgeon could not find the correct anatomical structures without the position and orientation information.

We also used glycerin as a flushing liquid instead of blood plasma because of the restriction on the experiment time. The glycerin liquid was warmed to maintain the swine's body temperature at a constant level so that no shocks were felt by the heart. The flushing speed during the experiment was $3-5 \mathrm{ml} / \mathrm{s}$.

The screenshots from the video obtained during the off-pump observation are shown in Figure 5. The image on the left is the normal image that corresponds to the case without flushing - we can only observe blood flow. The image at the center shows the chordae tendineae of the tricuspid valve, and the papillary muscle was also observed well for a few seconds. The image on the right clearly shows the leaflet of the tricuspid valve in the right atrium.

In these experiments, we successfully obtained very clear camera images of the interior of the beating heart during off-pump surgery.

\section{Discussion}

In in vitro experiments, we used real plasma, and the results were not very different from the in vivo results. However, some problems related to crystallization of the plasma were encountered, and we need to consider the temperature control.

The in vitro experiment was performed in the stationary condition, and the experimental results showed a trade-off between the observable depth and observable area.
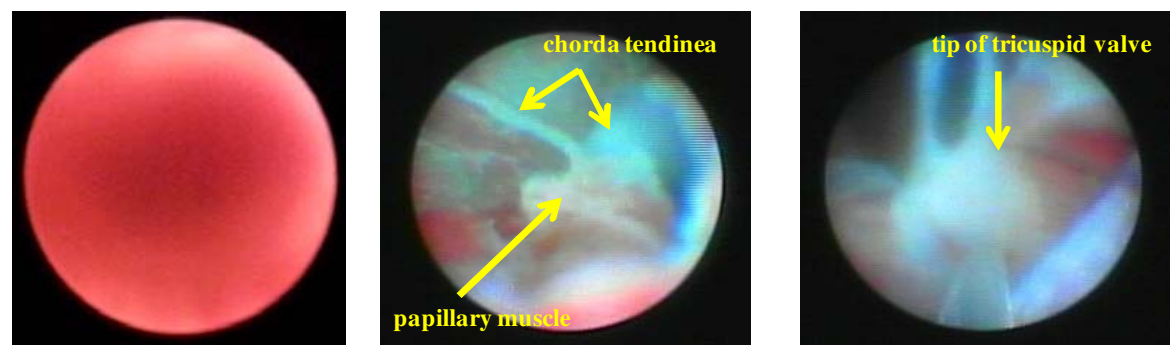

Fig. 5. In-vivo experiment: (Left) Observation without flushing-we observe nothing because of the presence of blood; (Center) Observation when flushing was performed-Chorda tendinea and papillary muscle were observed; (Right) The tip of the tricuspid valve was observed 


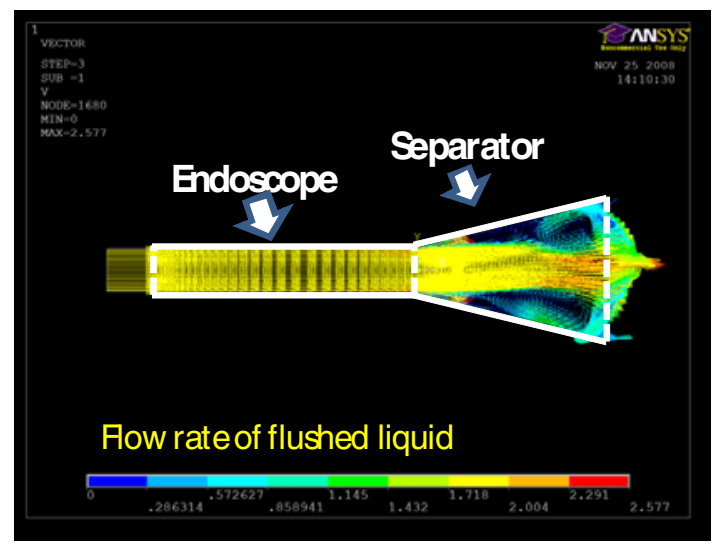

Fig. 6. $2 \mathrm{D}$ flow simulation of the device performed using ANSYS: the white lines represent the boundary, and the bar to the bottom represents the flow rate of the flushing liquid

We believe that this trade-off arises because the observable volume is constant for any flow rate while the observable depth varies with flow rate.

However, an actual clinical case involves the dynamic state, and further analysis is required for detailed design of the device. In a preliminary study, we attempted to perform 2D flow simulations using ANSYS CFD, as shown in Figure 6. In this simulation, the turbulent separated flow inside the separator flows along the separator wall, which is a boundary. Further, it is expected that in this simulation, it is possible to obtain a wider transparent volume for a high-velocity flow at the device tip. However, it is very difficult for us to perform an analysis by simulating the $3 \mathrm{D}$ incompressible unsteady flow. As the next step, we should carry out both parameter acquisitions by performing in vivo experiments and further high-cost simulations.

In the in vivo experiment, we developed a PC system that can be used to control the pumping timing through a manual switch or ECG signals; the system can also be used to store the endoscope images in real-time and periodically display images (similar to a stroboscopic view) on the monitor in order to maintain a clear view that is not distorted by the presence of blood cells. However, because of the time delay in the liquid flushing, it was impossible to control the timing of the flushing in the in vivo experiment. The flush pumping mechanism requires a few improvements before it is used in the next task.

In all cases, we successfully obtained clear endoscope images of the interior of the heart, even though each observation lasted for a short time. The next task is to combine surgical tools to perform the operation. N Suzuki has already developed the same kind of endoscope with a small robot; the endoscope can be used for intravascular operation[5].

\section{Conclusion}

In this study, we developed a new endoscope system to acquire clear endoscopic images of the interiors of blood vessels or the heart. These images are useful when 
performing simple procedures during cardiac surgery. By using this device, we can obtain high-resolution images to observe the inner structure of the heart and its pumping activity. Thus, we can perform better diagnosis and administer more effective therapy by applying robotic technologies inside the heart.

We are currently developing a small manipulator that we will combine with the observation device for performing advanced minimal invasive surgery in the near future.

This research is supported by MEXT Grant-in-Aid for Scientific Research (S) (\#17100008).

\section{References}

1. Timek, T.A., Nielsen, S.L., Lai, D.T., Tibaya, F., Liang, D., Daughters, G.T., Beineke, P., Hastie, T., Ingels Jr., N.B., Miller, D. C.: Mitral Annular Size Predicts Alfieri Stitch Tension in Mitral Edge-to-Edge Repair. J. Heart Valve Dis. 13(2), 165-173 (2004)

2. http: / /www. evalveinc. com/index.html (March 2009)

3. Linte, C.A., Wiles, A.D., Moore, N.H.J., Wedlake, C., Gerard, G., Jones, D., Bainbridge, D., Peters, T.M.: An augmented reality environment for image-guidance of off-pump mitral valve implantation. In: Proc. SPIE International Symposium on Medical Imaging 2007, San Diego, CA, February 17-22, 2007, pp. 6509-6522 (2007)

4. Li, M., Mazilu, D., Horvath, K.A.: Robotic System for Transapical Aortic Valve Replacement with MRI Guidance. In: Metaxas, D., Axel, L., Fichtinger, G., Székely, G. (eds.) MICCAI 2008, Part II. LNCS, vol. 5242, pp. 476-484. Springer, Heidelberg (2008)

5. Suzuki, N., Hattori, A., Suzuki, S., Otake, Y.: Development of a surgical robot system for endovascular surgery with augmented reality function. In: Medicine Meets Virtual Reality, vol. 15, pp. 460-463. IOS Press, Amsterdam (2007)

6. Masamune, K., Mizutani, M., Tsukihara, H., Takamoto, S., Matsumiya, K., Dohi, T.: A new blood-permeable endoscope system with plasma flushing for off-pump cardiac surgery. International Journal of Computer Assisted Radiology and Surgery 3(suppl. 1), S245-S246 (2008) 